ARTICLE

Received 29 Nov 2012 | Accepted 26 May 2013 | Published 26 Jun $2013 \quad$ DOI: 10.1038/ncomms3063

\title{
Magnetoelectric control of frozen state in a toroidal glass
}

\author{
Y. Yamaguchi ${ }^{1} \&$ T. Kimura ${ }^{1}$
}

The glass state of matter represents a frozen state of an atomically disordered system with local order only. Instead of atoms, systems with glassy states of magnetic and electric dipole moments in solids are known as spin and dipole glasses, respectively. In these conventional glasses, slow dynamics, such as relaxation and memory phenomena, are characteristics of their magnetic/dielectric properties. Here we propose a new glassy state in solids, a 'toroidal glass', in which toroidal moments-vector-like electromagnetic multipole moments breaking both space inversion and time reversal symmetries, and producing a linear magnetoelectric coupling-are randomly oriented and frozen. We investigate the dynamics of a linear magnetoelectric effect in $\mathrm{Ni}_{0.4} \mathrm{Mn}_{0.6} \mathrm{TiO}_{3}$ and find that the magnetoelectric responses strongly depend on the magnetoelectric cooling history and show striking memory effects. These unusual magnetoelectric dynamical features can be explained in the framework of a toroidal glass in which the toroidal frozen state can be controlled magnetoelectrically.

\footnotetext{
${ }^{1}$ Division of Materials Physics, Graduate School of Engineering Science, Osaka University, Toyonaka, Osaka 560-8531, Japan. Correspondence and requests for materials should be addressed to T.K. (email: kimura@mp.es.osaka-u.ac.jp).
} 
$\mathrm{T}$ he magnetoelectric (ME) effect, that is, the induction of either magnetization $M$ by an electric field $E\left(\mathrm{ME}_{E}\right.$ effect) or electric polarization $P$ by a magnetic field $H\left(\mathrm{ME}_{H}\right.$ effect), is a sensitive probe of the magnetic symmetry of magnetic insulators ${ }^{1-3}$. The terms of the free energy $F$ to be considered are $F=-M H-P E-\alpha H E-\beta H H E-\gamma H E E \ldots$. Here the first and second terms are the Zeeman and Stark energies, respectively. The third and fourth (fifth) terms give the linear and quadratic ME effects in which $P(M)$, linear or quadratic, in the applied field strength is induced by $H(E)$, respectively. On the basis of symmetry analysis, the linear effect $(P=\alpha H$ and $M=\alpha E$ with non-zero linear ME coefficient $\alpha$ ) is allowed only in magnetic insulators in which both space inversion and time reversal symmetries are broken. The toroidal moment $\mathbf{t}$ can be generated either by persistent orbital currents or certain spin orderings ${ }^{4,5}$. In the latter case, $\mathbf{t}$ is known as a multi-spin variable breaking both space inversion and time reversal symmetries, and is defined as $\mathbf{t} \propto \Sigma_{i} \mathbf{r}_{i} \times \mathbf{S}_{i}$, where $\mathbf{r}_{i}$ denotes the location of spin $S_{i}$ (refs 4,5). When $\mathbf{t}$ is non-zero, an off-diagonal and antisymmetric linear ME effect is allowed, that is, $\mathbf{P} \propto-\mathbf{t} \times \mathbf{H}$ and $\mathbf{M} \propto \mathbf{t} \times \mathbf{E}$, which are obtained from the free energy expansion analysis 6,7 . Thus, magnetic insulators showing a ferrotoroidic order (for example, $\mathrm{Ga}_{2-x} \mathrm{Fe}_{x} \mathrm{O}_{3}$ and $\mathrm{LiCoPO}_{4}$ ) can exhibit the antisymmetric linear ME effect ${ }^{8-10}$.

Recently, an $X Y$-like spin glass $\mathrm{Ni}_{x} \mathrm{Mn}_{1-x} \mathrm{TiO}_{3}(x \approx 0.42)^{11,12}$ was found to show the off-diagonal and antisymmetric linear $\mathrm{ME}_{H}$ effect ${ }^{13}$, although a linear ME effect is rarely seen in magnetically disordered systems, such as spin glasses. (A higherorder $\mathrm{ME}$ effect is seen in perovskite $(\mathrm{Sr}, \mathrm{Mn}) \mathrm{TiO}_{3}$, so-called 'multiglass', in which dipole and spin glass states coexist ${ }^{14}$.) In $\mathrm{Ni}_{x} \mathrm{Mn}_{1-x} \mathrm{TiO}_{3}$ with the ilmenite structure (centrosymmetric space group $R \overline{3})$, a magnetic $(\mathrm{Ni}, \mathrm{Mn})$ plane and a nonmagnetic Ti plane are alternately stacked along the hexagonal $c$ axis. The $\mathrm{Ni}^{2+}$ and $\mathrm{Mn}^{2+}$ ions are randomly distributed in the $(\mathrm{Ni}, \mathrm{Mn})$ plane and form a honeycomb lattice (inset of Fig. 1b). $\mathrm{Ni}_{0.4} \mathrm{Mn}_{0.6} \mathrm{TiO}_{3}$ (hereafter NMTO) exhibits successive spin glass transitions at $T_{\mathrm{SG} 1} \approx 9.5 \mathrm{~K}$ and $T_{\mathrm{SG} 2} \approx 6 \mathrm{~K}^{11,13}$, and is classified as an $X Y$-like spin glass with an easy-plane anisotropy ${ }^{15}$. The recently discovered antisymmetric linear $\mathrm{ME}_{H}$ effect in NMTO possesses ME tensor components $\alpha_{12}=-\alpha_{21} \neq 0$ below about $9 \mathrm{~K}^{13}$. The $\mathrm{ME}_{H}$ effect was reasonably explained by considering that the presence of the cross-product of $E$ and $H,(\mathbf{E} \times \mathbf{H})$, during the spin-freezing process polarizes net $t$ in the direction perpendicular to the honeycomb lattices. Thus, NMTO is a rare example showing that disordered frozen spins purely induce a linear ME coupling. This study examines cooling history and dynamical features, which are the most intriguing characteristics of the glassy frozen states, in the ME effect of NMTO and provide a new concept, 'toroidal glass'.

\section{Results}

Cooling-condition dependence of ME effect. First, we examine the ME-cooling-condition dependence on $P$ induced by the $\mathrm{ME}_{H}$ effect in a single crystal of NMTO. Figure 1 shows temperature (T) profiles of $P$ parallel to the [1-10] direction $\left(P_{[1-10]}\right)$ at $\mu_{0} H=9 \mathrm{~T}$ along [110] $\left(H_{[110]}\right)$. This configuration allows measurement of the ME tensor components $\alpha_{12}$ (or $\alpha_{21}$ ). Before the respective measurements, the specimen was cooled from $20 \mathrm{~K}$ $\left(>T_{\mathrm{SG} 1}\right)$ to $2 \mathrm{~K}$ in finite $|\mathbf{E} \times \mathbf{H}|$ with selected $E$ along [1-10] $\left(E_{[1-10]}\right)$ and $H_{[110]}$ (ME field cooling). Then, $P_{[1-10]}$ was taken at $\mu_{0} H_{[110]}=9 \mathrm{~T}$ in the absence of $E_{[1-10]}$. All the data shown in Fig. 1a were taken after $\mathrm{ME}$ field cooling with $E_{[1-10]}=$ $1 \mathrm{MV} \mathrm{m}^{-1}$ and selected $H_{\text {11101. }}$. Below $9 \mathrm{~K}$, magnetoelectrically induced $P_{[1-10]}$ develops in all the data. It is noteworthy that the magnitude of $P_{[1-10]}$ strongly depends on the strength of cooling

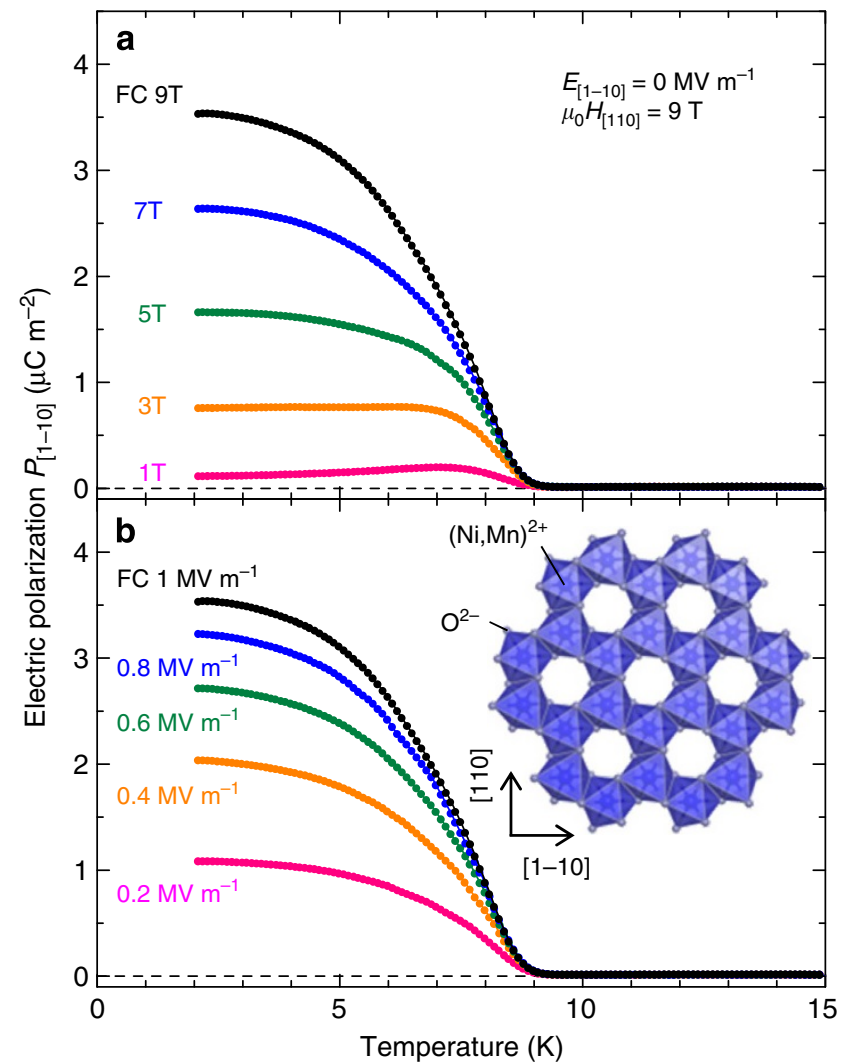

Figure 1 | ME-cooling-condition dependence on $\mathbf{P}$ induced by $\mathbf{M E}_{\boldsymbol{H}}$ effect in NMTO. $T$ dependence of $P_{[1-10]}$ measured at $\mu_{0} H_{[110]}=9 \mathrm{~T}$ and $E_{[1-10]}=0 \mathrm{MVm}^{-1}$ after several $\mathrm{ME}$ cooling procedures (a) at fixed $E_{[1-10]}=1 \mathrm{MVm}^{-1}$ and various $\mu_{0} H_{[110]}$ from 1 to $9 \mathrm{~T}$ (b) at various $E_{[1-10]}$ from 0.2 to $1 \mathrm{MV} \mathrm{m}^{-1}$ and $\mu_{0} H_{[110]}=9 \mathrm{~T}$. (For example, 'FC $1 \mathrm{MVm}^{-1}$, denotes the data $M E-$ cooled at $E_{[1-10]}=1 \mathrm{MVm}^{-1}$.) Inset of $\mathbf{b}$, crystal structure of NMTO projected along the hexagonal $c$ axis. Randomly distributed $\mathrm{Ni}^{2+}$ and $\mathrm{Mn}^{2+}$ ions form stacked honeycomb lattices.

$H_{[110]}$. Figure 1a clearly demonstrates that $P_{[1-10]}$ monotonically increases with increasing the cooling $H_{[110]}$. We also measured the cooling $E$ dependence of $P_{[1-10]}$. The results are shown in Fig. $1 \mathrm{~b}$. Before these measurements, $\mu_{0} H_{[110]}=9 \mathrm{~T}$ and selected $E_{[1-10]}$ were applied during the ME-field-cooling process. The magnitude of $P_{[1-10]}$ monotonously increases as the cooling $E_{[1-10]}$ increases. These results indicate that the magnitude of $P$ is very sensitive to both cooling $E$ and $H$, that is, ME-field-cooling condition. The observed remarkable cooling $H$ and $E$ effects on $P$ are reminiscent of the cooling $H$ effect on $M$ in spin glasses ${ }^{16}$ and/or the cooling $E$ effect on $P$ in dipole glasses ${ }^{17}$.

In ref. 13, it was proposed that the $\mathrm{ME}_{H}$ effect, that is, the induction of $P_{[1-10]}$ by applying $H_{[110]}$, observed in NMTO originates from the presence of net toroidal moment $t$ along [001] through the relation $\mathbf{P} \propto-\mathbf{t} \times \mathbf{H}$. If this scenario is correct, we can also expect the $\mathrm{ME}_{E}$ effect, the induction of $M$ along [110] $\left(M_{[110]}\right)$ induced by applying $E_{[1-10]}$, with the relation $\mathbf{M} \propto \mathbf{t} \times \mathbf{E}$ from phenomenological consideration ${ }^{7}$. Thus, we examined the $\mathrm{ME}_{E}$ effect in NMTO. Figure $2 \mathrm{~b}$ shows the time dependence of $M_{[110]}$ at $\mu_{0} H_{[110]}=0 \mathrm{~T}$ and $T=2 \mathrm{~K}$. Before the measurement, the ME cooling procedure was done with $\mu_{0} H_{[110]}=7 \mathrm{~T}$ and $E_{[1-10]}=1 \mathrm{MV} \mathrm{m}^{-1}$. As is common with spin glasses, a remnant magnetization caused by the magnetic-field cooling appears and shows a long-time relaxation ${ }^{16}$. During the relaxation process, various strengths of $E_{[1-10]}$ were applied, as plotted in Fig. 2a. Comparing Fig. 2a,b it is evident that the magnitude of $M_{[110]}$ 
systematically changes, depending on the strength and sign of applied $E_{[1-10]}$. Such an effect of $E_{[1-10]}$ on $M_{[110]}$ was not observed without the ME-field-cooling process. This result clearly confirms the presence of the $\mathrm{ME}_{E}$ effect in NMTO.

Figure 3a displays $E_{[1-10]}$ profiles of the change in remanent magnetization $\left[\Delta M_{[110]}=M_{[110]}(E)-M_{[110]}(0)\right]$ measured at $\mu_{0} H_{[110]}=0 \mathrm{~T}$ and $T=2 \mathrm{~K}$. Before each measurement, the MEfield cooling was performed at various $E_{[1-10]}$ and $H_{[110]}$. As seen in Fig. 3a, $\Delta M_{[110]}$ shows the linear $E_{[1-10]}$ dependence, $\Delta M_{[110]}=\alpha_{\mathrm{m}} E_{[1-10]}$, and the sign of $\alpha_{\mathrm{m}}$ is reversed by changing the sign of the ME field $(\mathbf{E} \times \mathbf{H})$ upon cooling. (Compare solid and broken lines in the same colour.) In addition to the linear $E$ dependence, the magnitude of $\Delta M_{[110]}$ (or $\alpha_{\mathrm{m}}$ ) strongly depends on that of $(\mathbf{E} \times \mathbf{H})$ upon cooling. As $|\mathbf{E} \times \mathbf{H}|$ increases, $\alpha_{\mathrm{m}}$ monotonically increases. To compare the $\mathrm{ME}_{E}$ effect with the $\mathrm{ME}_{H}$ effect, we also show in Fig. $3 \mathrm{~b}$ the $H_{[110]}$ dependence of $P_{[1-10]}$ measured at $E_{[1-10]}=0 \mathrm{MV} \mathrm{m}^{-1}$ and $T=2 \mathrm{~K}$ after the selected preparatory ME cooling procedures. As seen in

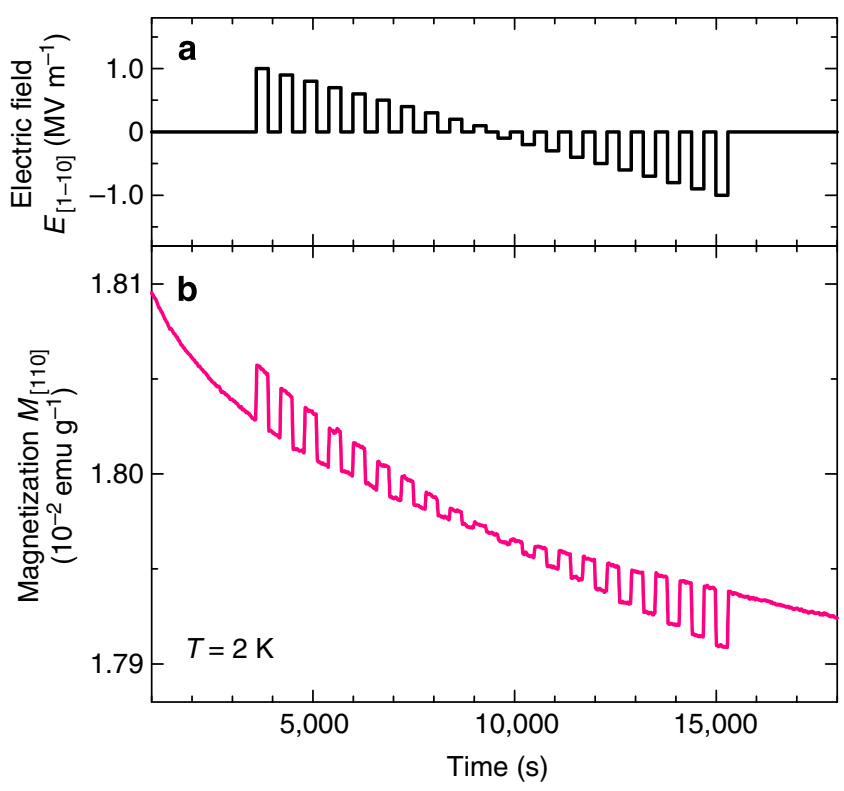

Figure $\mathbf{2}$ | Relaxation of $\boldsymbol{M}$ and $\mathbf{M E}_{\boldsymbol{E}}$ effect in $\mathbf{N M T O}$. Temporal evolution of (b) isothermal $M_{[110]}$ responding to (a) periodically applied $E_{[1-10]}$ at $T=2 \mathrm{~K}$ and $\mu_{0} H_{[110]}=0 \mathrm{~T}$. Before the measurement, ME cooling was done with $\mu_{0} H_{[110]}=7 \mathrm{~T}$ and $E_{[1-10]}=1 \mathrm{MVm}^{-1}$.
Fig. 3a,b, the $\mathrm{ME}_{H}$ and $\mathrm{ME}_{E}$ effects show a similar ME-fieldcooling dependence. The ME coefficient $\alpha_{\mathrm{p}}(=d P / d H)$ obtained from the $P-H$ curve cooled at $\mu_{0} H_{[110]}=7 \mathrm{~T}$ and $E_{[1-10]}=$ $1 \mathrm{MV} \mathrm{m}^{-1}$ is $\sim 0.2 \mathrm{ps} \mathrm{m}^{-1}$ at around $0 \mathrm{~T}$. Meanwhile, $\alpha_{\mathrm{m}}$

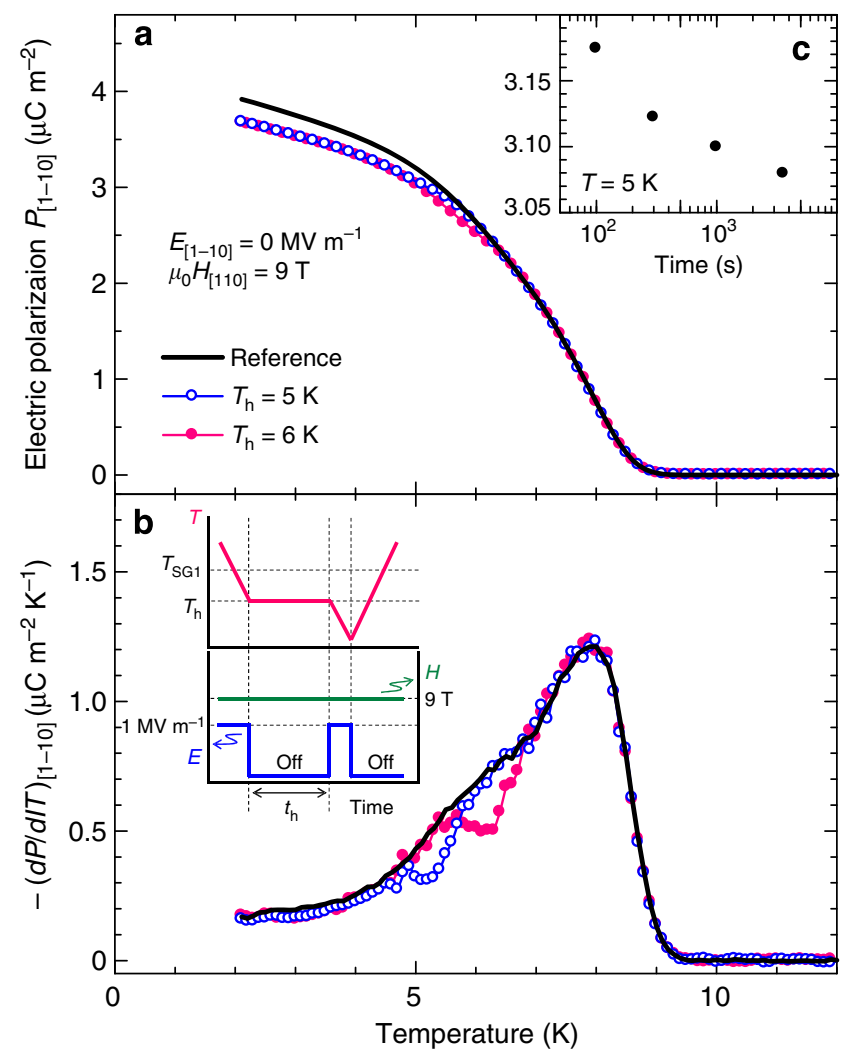

Figure 4 | ME memory effect in NMTO. Temperature dependence of (a) $P_{[1-10]}$ and $(\mathbf{b})-(d P / d T)_{[1-10]}$ taken upon warming at $\mu_{0} H_{[110]}=9 \mathrm{~T}$ and $E_{[1-10]}=0 \mathrm{MVm}^{-1}$. The solid black line shows a reference curve taken after continuous field cooling at $\mu_{0} H_{[110]}=9 \mathrm{~T}$ and $E_{[1-10]}=1 \mathrm{MVm}^{-1}$. The open blue circles and closed red circles were measured after a halt at $T_{\mathrm{h}}=5 \mathrm{~K}$ and $6 \mathrm{~K}$, respectively, during the same field-cooling procedure. During the halt time, $E_{[1-10]}$ was turned off. (c) The $t_{\mathrm{h}}$ dependence of $P_{[1-10]}$ measured at $5 \mathrm{~K}$ for $T_{\mathrm{h}}=6 \mathrm{~K}$. Inset of $\mathbf{b}$, schematic representation of field-cooling and warming procedure, including a halt. The upper panel shows the temporal evolution of $T$, and the lower panel shows those of $E$ and $H$ during a ME cooling and a measurement.

\section{a}

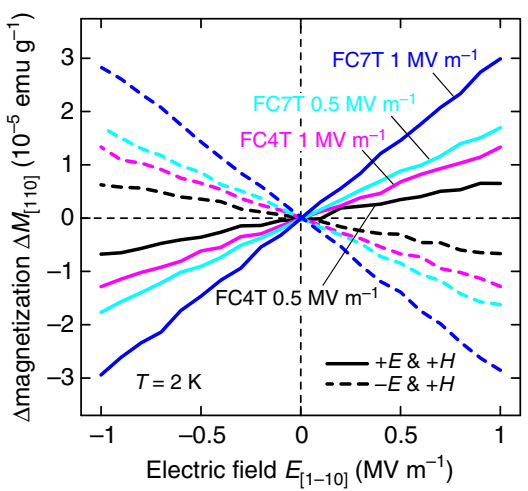

b

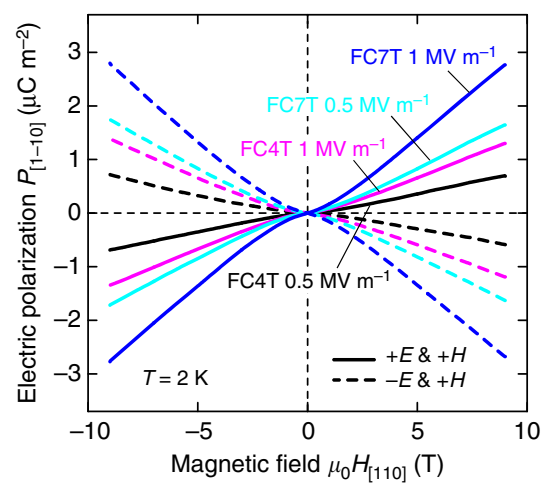

Figure 3 | Comparison of $\mathbf{M E}_{\mathbf{E}}$ and $\mathbf{M E}_{\boldsymbol{H}}$ effect in $\mathbf{N M T O}$. (a) $E_{[1-10]}$ dependence of $\Delta M_{[110]}=M_{[110]}(E)-M_{[110]}(0)$ and $(\mathbf{b}) H_{[110]}$ dependence of $P_{[1-10]}$ at $T=2 \mathrm{~K}$ after $\mathrm{ME}$ cooling with selected $E_{[1-10]}$ and $H_{[110]}$. (For example, 'FC7T $1 \mathrm{MVm}^{-1}$, denotes the data $\mathrm{ME}-$ cooled at $\mu_{0} H_{[110]}=7 \mathrm{~T}$ and $E_{[1-10]}=1 \mathrm{MVm}^{-1}$.) Solid and broken lines represent the data taken after $\mathrm{ME}$ cooling with $\left(+E_{,}+H\right)$ and $\left(-E_{,}+H\right)$, respectively. 
obtained from the $M-E$ curve for the same ME-field-cooling condition is $\sim 0.2 \mathrm{ps} \mathrm{m}^{-1}$. This good agreement indicates that the origin of the $\mathrm{ME}_{H}$ and $\mathrm{ME}_{E}$ effects is the same, and supports the presence of net $\mathbf{t}^{13}$.

ME memory effect. We also found that NMTO shows the ME version of 'memory effect', which is common in glassy systems ${ }^{18-21}$. First, we took $P_{[1-10]}$ by a usual measurement process: $P_{[1-10]}$ was taken upon warming at $\mu_{0} H_{[110]}=9 \mathrm{~T}$ and $E_{[1-10]}=0 \mathrm{MVm}^{-1}$ after $\mathrm{ME}$ cooling at $\mu_{0} H_{[110]}=9 \mathrm{~T}$ and $E_{[1-10]}=1 \mathrm{MV} \mathrm{m}^{-1}$. The obtained $P_{[1-10]}$ is referred to as the reference curve $\left(P_{\text {ref }}\right)$ (a black solid curve in Fig. $4 a$ ). Then, we again measured $P_{[1-10]}$ by the same procedure, except for including a halt for $t_{\mathrm{h}}=3,600 \mathrm{~s}$ at an intermediate temperature $T_{\mathrm{h}}(=6 \mathrm{~K}$ or $5 \mathrm{~K})$ during cooling. During the halt, $E_{[1-10]}$ was turned off. The schematic measurement protocol is illustrated in the inset of Fig. $4 \mathrm{~b}$. For all the measurements, the cooling and warming rate is constant at $1 \mathrm{~K} \mathrm{~min}^{-1}$. As seen in Fig. $4 \mathrm{a}, P_{[1-10]}$ after a halt on cooling $\left(P_{\mathrm{h}}\right)$ slightly decreases compared with $P_{\text {ref }}$ below $T_{\mathrm{h}}$. A more distinct difference is observed in between $-d P_{\text {ref }} / d T$ and $-d P_{\mathrm{h}} / d T$ (see Fig. $4 \mathrm{~b}$ ). The $T$ dependence of $-d P_{\mathrm{h}} / d T$ with $T_{\mathrm{h}}=6 \mathrm{~K}$ shows a 'dip'-like structure around $T_{\mathrm{h}}=6 \mathrm{~K}$, and $-d P_{\mathrm{h}} / d T$ almost overlaps $-d P_{\text {ref }} / d T$ below $5.5 \mathrm{~K}$ and above $7 \mathrm{~K}$. In the data of $T_{\mathrm{h}}=5 \mathrm{~K},-d P_{\mathrm{h}} / d T$ clearly shows a dip around $T_{\mathrm{h}}=5 \mathrm{~K}$. We also measured $t_{\mathrm{h}}$ dependence of $P_{[1-10]}$. As seen in Fig. $4 \mathrm{c}, P_{[1-10]}$ measured at $5 \mathrm{~K}$ for $T_{\mathrm{h}}=6 \mathrm{~K}$ shows a slow decay over $t_{\mathrm{h}}=1 \mathrm{~h}$. These results clearly indicate that the long-time relaxation of $P_{[1-10]}$ occurs during the halts and NMTO memorizes the halt during cooling. Thus, we successfully observed the slow dynamics and memory effect by use of ME effect for the first time.

\section{Discussion}

Let us discuss the cooling- $(\mathbf{E} \times \mathbf{H})$ dependence of the ME effect in NMTO. According to the consideration of the free energy in a magnetic system in which spin ordering breaks the time reversal and space inversion symmetries, the free energy related to the ME effect is expressed as $F_{\mathrm{ME}}=-a(\mathbf{E} \cdot \mathbf{H})-\mathbf{t} \cdot[\mathbf{E} \times \mathbf{H}] \ldots$, where the pseudoscalar $a$ is coupled to the divergence of magnetic field, and is zero in the absence of magnetic monopoles ${ }^{7}$. This means that the free energy is stabilized by the presence of $(\mathbf{E} \times \mathbf{H})$ and a toroidal moment $\mathbf{t}$ along $(\mathbf{E} \times \mathbf{H})$. Meanwhile, in spin glass systems with replica symmetry breaking ${ }^{22}$, multi-valley structures in their free energies are considered to develop and be responsible for their characteristics, such as slow relaxations, dependence on initial conditions and remanent effects. Thus, it is reasonable to consider that one of the multi-valley states having net $\mathbf{t}$ is stabilized due to the presence of a certain $(\mathbf{E} \times \mathbf{H})$ during the spin-freezing process and that the change in the magnitude of $(\mathbf{E} \times \mathbf{H})$ during the freezing makes the system into a different valley state with a different net $\mathbf{t}$.

Figure $5 a-c$ depicts proposed schematic spin configurations of the spin-frozen states after ME cooling processes at zero, low and high $\mathrm{ME}$ fields $(\mathbf{E} \times \mathbf{H})$, respectively. Free energy related with toroidal moment $\mathbf{t}$ can be expressed as $F_{\mathrm{t}}=-\mathbf{t} \cdot(\mathbf{E} \times \mathbf{H})^{7}$. Thus, when $|\mathbf{E} \times \mathbf{H}|$ is absent upon cooling, there is no energy gain by the presence of $\mathbf{t}$, and then no net $\mathbf{t}$ develops, which means spins with an easy-plane anisotropy are randomly oriented within the plane (Fig. 5a). However, by cooling the system in the finite ME fields $|\mathbf{E} \times \mathbf{H}|$, the induction of net $\mathbf{t}$ gives rise to energy gain (Fig. 5b). As $|\mathbf{E} \times \mathbf{H}|$ upon cooling increases, freezing spins are gradually oriented in a vortex-like manner, each local $\mathbf{t}$ polarized along the same direction, and then net $\mathbf{t}$ increases (Fig. 5c). This scenario well explains the observed ME-field-cooling dependence of $\mathrm{ME}_{E}$ and $\mathrm{ME}_{H}$ effects (Fig. 3). Figure 5d,e display contour plots of the magnitude of $P_{[1-10]}$ and $\Delta M_{[110]}$ versus the strength of $E_{[1-10]}$ and $H_{[110]}$ upon cooling, respectively. Both $\Delta M_{[110]}$ and $P_{[1-10]}$, that is, $\alpha_{\mathrm{m}}$ and $\alpha_{\mathrm{p}}$, monotonically increase with increasing $|\mathbf{E} \times \mathbf{H}|$. These plots further confirm the above scenario and demonstrate ME control of the spin-frozen state (or net $\mathbf{t}$ ) by changing $|\mathbf{E} \times \mathbf{H}|$. In addition, the observed ME-field-cooling dependence indicates that there are a number of metastable toroidal frozen states. Such huge degeneracy is discussed as the multi-valley structure in free energy for conventional spin glasses with replica symmetry breaking ${ }^{22}$ and can be observed as the cooling $H$ dependence of $M^{16,23}$. In other words, toroidal moments in NMTO appear to behave as glass. Furthermore, the ME slow dynamics, as observed for the a

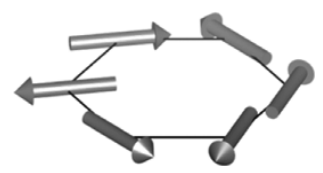

b

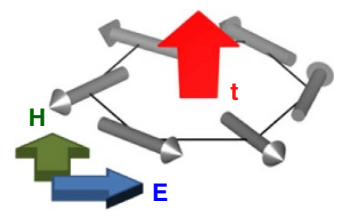

C

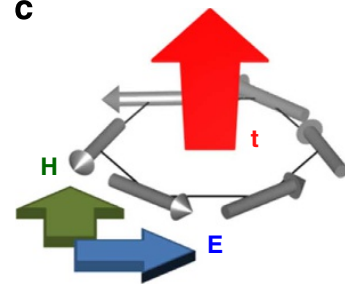

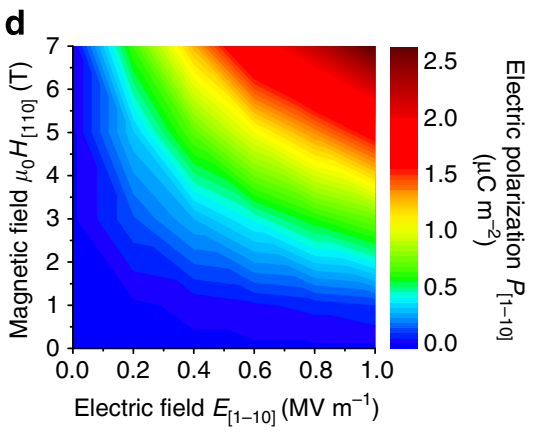

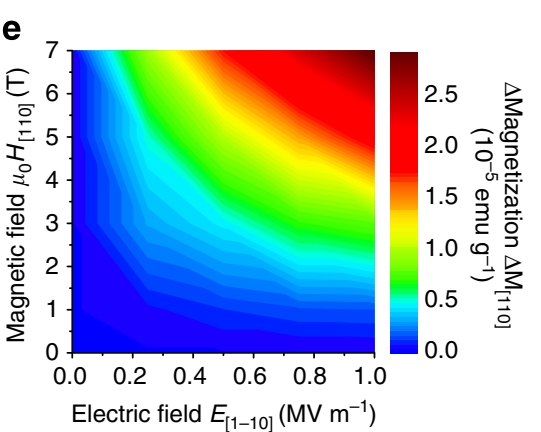

Figure 5 | Toroidal frozen state controlled magnetoelectrically. (a-c) Proposed relationships among the strength of ME cooling field $(\mathbf{E} \times \mathbf{H})$, the induced spin (gray arrow) configurations and the concomitant toroidal moment $\mathbf{t}$ in the spin-frozen state for NMTO with the honeycomb lattice. (d) Contour plot of $P_{[1-10]}$ measured at $T=2 \mathrm{~K}, \mu_{0} H_{[110]}=9 \mathrm{~T}$, and $E_{[1-10]}=0 \mathrm{MVm}^{-1}$ versus $E_{[1-10]}$ and $H_{[110]}$ upon the ME-field-cooling process. (e) Counter plot of $\Delta M_{[110]}$ at $T=2 \mathrm{~K}, \mu_{0} H_{[110]}=0 \mathrm{~T}$, and $E_{[1-10]}=1 \mathrm{MVm}^{-1}$ versus $E_{[1-10]}$ and $H_{[110]}$ upon $\mathrm{ME}$-field-cooling process. 
memory effect, strongly suggest the validity of the glass nature of toroidal moment.

When looking at the above-mentioned ME-related free energy $F_{\mathrm{ME}}$, the development of net the pseudoscalar $a$, which is related to magnetic monopoles, can also be expected in the presence of $(\mathbf{E} \cdot \mathbf{H})$. However, we did not observe any ME signals after the ME-field-cooling only with finite $(\mathbf{E} \cdot \mathbf{H})$. Hence, such a spinfrozen state may not exist in the present system, although we do not have a clear reason at this time.

Thus, we conclude that there exists a novel glass state in solids, termed 'toroidal glass', which is regarded as the glass of the fourth ordered form, '(anti)ferrotoroidic ordered state ${ }^{\text {, }, 7}$. Measurements of ME-cooling-condition dependence of magnetochiral dichroism, non-reciprocal X-ray gyrotropy and magnetic diffuse scattering can be other complementary methods to verify the toroidal glass state ${ }^{7,24}$. Other $X Y$-like spin glass systems (for example, $\mathrm{BaCo}_{6} \mathrm{Ti}_{6} \mathrm{O}_{19}$ (ref. 25)) can be promising candidates having the toroidal glass state.

\section{Methods}

Crystal growth. A single crystal of NMTO was grown by the floating zone method, as reported previously ${ }^{26}$. First, a polycrystalline feed rod was prepared by a solid-state reaction. Stoichiometric quantities of $\mathrm{NiO}, \mathrm{Mn}_{3} \mathrm{O}_{4}$ and $\mathrm{TiO}_{2}$ powders with $99.9 \%$ purity were mixed and heated at $1,000{ }^{\circ} \mathrm{C}$ for $10 \mathrm{~h}$ in air. The resulting polycrystalline sample was ground into powders again, and then pressed into a rod with the dimension of about $6 \mathrm{~mm}$ in diameter and $100 \mathrm{~mm}$ in length. The rod was sintered again at $1,100^{\circ} \mathrm{C}$ for $15 \mathrm{~h}$ in air. The crystal growth was carried out on the sintered rod with the use of a halogen-lamp image furnace in flowing air at a rate of $500 \mathrm{ml} \mathrm{min}-1$. The growth rate was $1-3 \mathrm{~mm} \mathrm{~h}^{-1}$. Thus, a single crystal with clear facets parallel to the $c$-plane (hexagonal setting) was obtained.

Measurements of ME effects. The obtained crystal was oriented with use of Laue $\mathrm{X}$-ray diffraction patterns and was cut into two rectangular plates with the widest faces parallel to the (1-10) plane. The dimensions of these specimens were $17.5 \mathrm{~mm}^{2} \times 0.1 \mathrm{~mm}$ and $18.5 \mathrm{~mm}^{2} \times 0.4 \mathrm{~mm}$ for measurements of electric polarization $(P)$ and magnetization $(M)$, respectively. Silver electrodes were vacuumdeposited onto the widest faces of these specimens. To obtain $P$ parallel to [1-10] $\left(P_{[1-10]}\right)$ as functions of temperature $(T)$ and magnetic field $(H)$ applied along [110] $\left(H_{[110]}\right)$, pyroelectric and ME currents were measured during $T$ - and $H$-sweeping procedures, respectively, in a superconducting magnet (Quantum Design, PPMS) by using an electrometer (Keithley Instruments 6517A). To measure $M$ along [110] $\left(M_{[110]}\right)$ as a function of electric field $(E)$ applied along [1-10] $\left(E_{[1-10]}\right)$, a homemade insert, which allows the application of $E$ during $M$ measurements was installed in a magnetometer (Quantum Design, MPMS SQUID VSM). On the home-made insert, the silver electrodes vacuum-deposited onto the sample were electrically connected with an external voltage source (Keithley Instruments $6517 \mathrm{~A}$ ) via copper wires. All the ME measurements were done after ME-fieldcooling procedures at selected $E_{[1-10]}$ and $H_{[110]}$

\section{References}

1. Birss, R. R. Symmetry and Magnetism North-Holland, Amsterdam, 1966).

2. O'Dell, T. H. The Electrodynamics of Magneto-Electric Media (North-Holland, Amsterdam, 1970).

3. Fiebig, M. Revival of the magnetoelectric effect. J. Phys. D Appl. Phys. 38, R123-R152 (2005).

4. Dubovik, V. M. \& Tosunyan, L. A. Toroidal moments in the physics of electromagnetic and weak interactions. Sov. J. Part. Nucl. 14, 504-519 (1983).

5. Ginzburg, V. L., Gorbatsevich, A. A., Kopayev, Y. V. \& Volkov, B. A. On the problem of superdiamagnetism. Solid State Commun. 50, 339-343 (1984).

6. Schmid, H. On ferrotoroidics and electrotoroidic, magnetotoroidic and piezotoroidic effects. Ferroelectrics 252, 41-50 (2001).

7. Spaldin, N. A., Fiebig, M. \& Mostovoy, M. The toroidal moment in condensedmatter physics and its relation to the magnetoelectric effect. J. Phys. Condens. Matter 20, 434203 (2008).
8. Popov, Y. F. et al. Magnetoelectric effect and toroidal ordering in $\mathrm{Ga}_{2-x} \mathrm{Fe}_{x} \mathrm{O}_{3}$. J. Exp. Theor. Phys. 87, 146-151 (1998).

9. Kornev, I. et al. Magnetoelectric properties of $\mathrm{LiCoPO}_{4}$ and $\mathrm{LiNiPO}_{4}$. Phys. Rev. B 62, 12247-12253 (2000).

10. Van Aken, B. B., Rivera, J.-P., Schmid, H. \& Fiebig, M. Observation of ferrotoroidic domains. Nature 449, 702-705 (2007).

11. Ito, A., Kawano, H., Yoshizawa, H. \& Motoya, K. Magnetic properties and phase diagram of $\mathrm{Ni}_{x} \mathrm{Mn}_{1-x} \mathrm{TiO}_{3}$. J. Magn. Magn. Mater. 104-107, 1637-1638 (1992).

12. Kawano, H., Yoshizawa, H., Ito, A. \& Motoya, K. Two successive transitions in nondiluted Heisenberg-like spin glass $\mathrm{Ni}_{0.42} \mathrm{Mn}_{0.58} \mathrm{TiO}_{3}$. J. Phys. Soc. Jpn 62, 2575-2578 (1993).

13. Yamaguchi, Y., Nakano, T., Nozue, Y. \& Kimura, T. Magnetoelectric effect in an $X Y$-like spin glass system $\mathrm{Ni}_{x} \mathrm{Mn}_{1-x} \mathrm{TiO}_{3}$. Phys. Rev. Lett. 108, 057203 (2012).

14. Shvartsman, V. V., Bedanta, S., Borisov, P., Kleemann, W., Tkach, A. \& Vilarinho, P. M. Sr,Mn) $\mathrm{TiO}_{3}$ : a magnetoelectric multiglass. Phys. Rev. Lett. 101, 165704 (2008).

15. Kawamura, H. The ordering of XY spin glasses. J. Phys. Condens. Matter 23, 164210 (2011).

16. Binder, K. \& Young, A. P. Spin-glasses: experimental facts, theoretical concepts, and open questions. Rev. Mod. Phys. 58, 801-976 (1986).

17. Vugmeister, B. E. \& Glinchuk, M. D. Dipole glass and ferroelectricity in random-site electric dipole systems. Rev. Mod. Phys. 62, 993-1026 (1990).

18. Jonason, K., Vincent, E., Hammann, J., Bouchaud, J. P. \& Nordblad, P. Memory and chaos effects in spin glasses. Phys. Rev. Lett. 81, 3243-3246 (1998).

19. Jönsson, P. E., Mathieu, R., Nordblad, P., Yoshino, H., Aruga Katori, H. \& Ito, A. Nonequilibrium dynamics of spin glasses: examination of the ghost domain scenario. Phys. Rev. B 70, 174402 (2004).

20. Sasaki, M., Jönsson, P. E., Takayama, H. \& Mamiya, H. Aging and memory effects in superparamagnets and superspin glasses. Phys. Rev. B 71, 104405 (2005).

21. Colla, E. V., Chao, L. K., Weissman, M. B. \& Viehland, D. D. Aging in a relaxor ferroelectric: scaling and memory effect. Phys. Rev. Lett. 85, 3033-3036 (2000).

22. Mézard, M., Parisi, G., Sourlas, N., Toulouse, G. \& Virasoro, M. Nature of the spin-glass phase. Phys. Rev. Lett. 52, 1156-1159 (1984).

23. Lundgren, L., Svedlindh, P., Nordblad, P. \& Beckman, O. Dynamics of the relaxation-time spectrum in a CuMn spin-glass. Phys. Rev. Lett. 51, 911-914 (1983).

24. Manuel, P., Chapon, L. C., Radaelli, P. G., Zheng, H. \& Mitchell, J. F. Magnetic correlations in the extended Kagome $\mathrm{YBaCo}_{4} \mathrm{O}_{7}$ probed by single-crystal neutron scattering. Phys. Rev. Lett. 103, 037202 (2009).

25. Batlle, X., Labarta, A., Martínez, B., Obrador, X., Cabañas, V. \& Vallet-Regí, M. Spin glass transition in $\mathrm{BaCo}_{6} \mathrm{Ti}_{6} \mathrm{O}_{19}$. J. Appl. Phys. 70, 6172-6174 (1991).

26. Takei, H. Preferentially orientated precipitation in $\mathrm{MnTiO}_{3}$ single crystals. J. Mater. Sci. 16, 1310-1316 (1981).

\section{Acknowledgements}

We thank T. Taniguchi, H. Kawamura and L. Chapon for fruitful discussions. This work was supported by KAKENHI (grant numbers 24244058 and 20001004) and the Global COE Program (programme number G10), MEXT, Japan.

\section{Author contributions}

Y.Y. and T.K. designed this work and wrote the paper. Y.Y. carried out sample preparation and characterization, as well as magnetoelectric measurements. T.K. directed the research.

\section{Additional information}

Competing financial interests: The authors declare no competing financial interests.

Reprints and permission information is available online at http://npg.nature.com/ reprintsandpermissions/

How to cite this article: Yamaguchi, Y. \& Kimura, T. Magnetoelectric control of frozen state in a toroidal glass. Nat. Commun. 4:2063 doi: 10.1038/ncomms3063 (2013). 